PROCEEDINGS OF THE

AMERICAN MATHEMATICAL SOCIETY

Volume 130, Number 2, Pages 549-553

S 0002-9939(01)06344-

Article electronically published on September 19, 2001

\title{
SHUFFLE THE PLANE
}

\author{
MIKLÓS ABÉRT AND TAMÁS KELETI
}

(Communicated by David Preiss)

\begin{abstract}
We prove that any permutation $p$ of the plane can be obtained as a composition of a fixed number (209) of simple transformations of the form $(x, y) \rightarrow(x, y+f(x))$ and $(x, y) \rightarrow(x+g(y), y)$, where $f$ and $g$ are arbitrary $\mathbb{R} \rightarrow \mathbb{R}$ functions.

As a corollary we get that the full symmetric group acting on a set of continuum cardinal is a product of finitely many (209) copies of two isomorphic Abelian subgroups.
\end{abstract}

We investigate what transformations of the plane we can get by (finitely many) vertical and horizontal "slides", which we define as follows.

Definition 1. By a vertical (resp. horizontal) slide we mean an $\mathbb{R}^{2} \rightarrow \mathbb{R}^{2}$ map of the form $(x, y) \rightarrow(x, y+f(x))$ (resp. $(x, y) \rightarrow(x+g(y), y))$, where $f$ (resp. $g$ ) is an arbitrary $\mathbb{R} \rightarrow \mathbb{R}$ function.

By a slide we shall always mean a vertical or horizontal slide.

Note that geometrically a vertical (resp. horizontal) slide means a transformation of the plane in which we translate vertical (resp. horizontal) lines vertically (resp. horizontally).

Clearly any slide is a permutation of the plane, so the question is which permutations we can get by using (finitely many) slides. One can also ask the following (weaker) question: When can a subset of the plane be transformed to another subset using (finitely many) slides? Clearly, the sets must have the same cardinality and their complements must have the same cardinality, too - so the question is whether these conditions are sufficient or there exist other invariants of these maps.

In this paper we answer these questions by proving the following result:

Theorem 2. Any permutation of the plane can be obtained by a fixed number (209) of slides. That is, for any permutation $p$ of the plane there exist $\mathbb{R} \rightarrow \mathbb{R}$ functions $f_{1}, \ldots, f_{105}$ and $g_{1}, \ldots, g_{104}$ such that we have $p=F_{1} G_{1} \ldots F_{104} G_{104} F_{105}$, where $F_{i}(x, y)=\left(x, y+f_{i}(x)\right)$ and $G_{i}(x, y)=\left(x+g_{i}(y), y\right)$.

Therefore the only invariants are the cardinality and the cardinality of the complement; a set can be mapped to another set by finitely many slides if and only if they have the same cardinality and their complements have the same cardinality,

Received by the editors July 11, 2000.

2000 Mathematics Subject Classification. Primary 26B40; Secondary 03E05, 20B30, 20 D40.

The research of the first author was supported by the Hungarian National Foundation for Scientific Research Grant 32325.

The research of the second author was supported by the Hungarian National Foundation for Scientific Research Grant T26176. 
too. In particular, there is no finitely additive non-negative function from the set of all subsets of the plane that agrees with ordinary area on squares and is invariant under both vertical and horizontal slides.

Since both the vertical and the horizontal slides form (isomorphic) Abelian subgroups of the group of all permutations of $\mathbb{R}^{2}$, we also get the following (purely group-theoretic) result:

Corollary 3. The full symmetric group acting on a set of continuum cardinal is a product of finitely many (209) copies of two isomorphic Abelian subgroups.

This is where the original motivation of this investigation comes from. In [1 the same result (excluding the constant 209) is proved for the full symmetric group acting on a countable set via the analogous result about slides on $\mathbb{Z} \times \mathbb{Z}$. Some ideas of the proof of Theorem 2 also come from the $\mathbb{Z} \times \mathbb{Z}$ proof of [1].

It is also proved in [1] that the full symmetric group acting on any set is a product of finitely many Abelian subgroups. There - in the non-trivial infinite case - three Abelian subgroups were used and one of them was non-isomorphic to the other two.

In order to make our proof more transparent we shall prove Theorem 2 via a lemma and several claims - the last one claims Theorem 2, At each claim we also state the number of slides we use. We never show the calculation of this number since it is straightforward using the obvious fact that the composition of two vertical (resp. horizontal) slides is just one vertical (resp. horizontal) slide.

Notation 4 . In the sequel $\mathbb{N}$ will denote the set of positive integers, $\operatorname{card}(A)$ the cardinality of the set $A, \mathfrak{c}$ the continuum cardinal and $\left.\phi\right|_{A}$ the restriction of the map $\phi$ to the set $A$.

Lemma 5. One can give a countable partition $\bigcup_{n=1}^{\infty} A_{n}=\mathbb{R}$ and continuum many translated copies of every $A_{n}$ such that the collection $\left\{A_{n}+t_{n, \alpha}: n \in \mathbb{N}, \alpha \in[0,1)\right\}$ of all translated copies are pairwise disjoint.

Proof. Let $H=\left\{h_{\alpha}: \alpha \in[0,1)\right\}$ be a Hamel basis (of $\mathbb{R}$ over $\mathbb{Q}$ ) such that $H \subset[0,1)$. For each $k \in \mathbb{Z}, l \in \mathbb{N} \cup\{0\}$ and $q_{1}, \ldots, q_{l} \in \mathbb{Q}$ let $A^{k, q_{1}, \ldots, q_{l}}$ contain the reals of $[k, k+1)$ of the form $q_{1} h_{\alpha_{1}}+\ldots+q_{l} h_{\alpha_{l}}$, where $h_{\alpha_{i}} \in H$ and $h_{\alpha_{1}}<\ldots<h_{\alpha_{l}}$. Clearly this is a countable partition of $\mathbb{R}$.

We shall prove that each $A^{k, q_{1}, \ldots, q_{l}}$ has continuum many pairwise disjoint translated copies in $[k, k+2]$. This will complete the proof since we can easily translate the countably many intervals of length 2 into disjoint intervals, which makes the collection of all translated copies pairwise disjoint.

For fixed $l, k, q_{1}, \ldots, q_{l}$, let $r \in \mathbb{Q} \cap[0,1)$ be distinct from $q_{1}, \ldots, q_{l}$ such that $q_{i}+r \neq q_{j}$ for any $i, j$. (The last condition is not necessary but it makes the following argument simpler.) We claim that the translated sets $A^{k, q_{1}, \ldots, q_{l}}+r h_{\alpha}$ $(\alpha \in[0,1))$ are pairwise disjoint. Indeed, expressing any $x \in A^{k, q_{1}, \ldots, q_{l}}+r h_{\alpha}$ according to the Hamel base $H$ there is exactly one term $q h_{\beta}$ with coefficient $q$ distinct from $q_{1}, \ldots, q_{l}$, which implies that $h_{\alpha}=h_{\beta}$, so $x$ cannot be in another translated set $A^{k, q_{1}, \ldots, q_{l}}+r h_{\alpha^{\prime}}$.

Claim 6. The horizontal line $e=\mathbb{R} \times\{0\}$ can be mapped to a set that contains the horizontal strip $S=\mathbb{R} \times[0,1)$ (by 3 slides).

Proof. We will map $S$ into $e$ by 3 slides; clearly the inverse of such a map has the required properties. 
Let $\bigcup_{n=1}^{\infty} A_{n}=\mathbb{R}$ be the partition and $\left\{t_{n, \alpha}: n \in \mathbb{N}, \alpha \in[0,1)\right\}$ the collection of translations given in Lemma 5 .

First, for every $n \in \mathbb{N}$ and $x \in A_{n}$ translate the line $\{x\} \times \mathbb{R}$ by $n$ (vertically). The image of $S$ is $S_{1}=\bigcup_{n=1}^{\infty} A_{n} \times[n, n+1)$.

Then for every $n \in \mathbb{N}$ and $\alpha \in[0,1)$ translate $\mathbb{R} \times\{n+\alpha\}$ by $t_{n, \alpha}$ (horizontally). The image of $S_{1}$ is $S_{2}=\bigcup_{n=1}^{\infty} \bigcup_{\alpha \in[0,1)}\left(A_{n}+t_{n, \alpha}\right) \times\{n+\alpha\}$. Since the sets $\left\{A_{n}+t_{n, \alpha}: n \in \mathbb{N}, \alpha \in[0,1)\right\}$ are pairwise disjoint we can map (in fact, project) $S_{2}$ into $e=\mathbb{R} \times\{0\}$ by a vertical slide.

Claim 7 . The half-strip $[0, \infty) \times[0,1)$ can be mapped to the half-plane $[0, \infty) \times \mathbb{R}$ (by 4 slides).

Proof. First, for each $n \in \mathbb{N}$ we translate the vertical strip $\left[\frac{n(n-1)}{2}, \frac{n(n+1)}{2}\right) \times \mathbb{R}$ by $n-1$ vertically, then for each $n \in \mathbb{N}$ the horizontal strip $\mathbb{R} \times[n-1, n)$ by $-\frac{n(n-1)}{2}$ horizontally, then (for each $m=0,1,2, \ldots$ ) the strip $[m, m+1) \times \mathbb{R}$ by $-2 m$ vertically and finally (for each $n \in \mathbb{N}$ ) the strip $\mathbb{R} \times[-n,-n+1$ ) by $-n$ horizontally.

This way the half-strip $[0, \infty) \times[0,1)$ first goes to $\bigcup_{n=1}^{\infty}\left[\frac{n(n-1)}{2}, \frac{n(n+1)}{2}\right) \times[n-1, n)$, then this set goes to $\bigcup_{n=1}^{\infty}[0, n) \times[n-1, n)=\bigcup_{m=0}^{\infty}[m, m+1) \times[m, \infty)$, which goes to $\bigcup_{m=0}^{\infty}[m, m+1) \times[-m, \infty)=[0, \infty] \times[0, \infty] \cup \bigcup_{n=1}^{\infty}[n, \infty) \times[-n,-n+1)$, so finally we get the half-plane $[0, \infty) \times \mathbb{R}$.

Claim 8. The set $\mathbb{R}^{2} \backslash e$ can be mapped into $e=\mathbb{R} \times\{0\}$ (by 11 slides).

Proof. Gluing together Claims 6 and 7 we can map $e$ onto a set that contains the half-plane $[0, \infty) \times \mathbb{R}$. Clearly, the same map maps $\mathbb{R}^{2} \backslash e$ into the half-plane $(-\infty, 0) \times \mathbb{R}$. The inverse of this map maps the half-plane $[0, \infty) \times \mathbb{R}$ into $e$, so (by symmetry) we can map $(-\infty, 0) \times \mathbb{R}$ into $e$, too. Doing this after the first map, $\mathbb{R}^{2} \backslash e$ goes into $e=\mathbb{R} \times\{0\}$.

Claim 9. Any subset $A$ of the plane with $\operatorname{card}(A)=\mathfrak{c}$ can be mapped to a set that contains $e=\mathbb{R} \times\{0\}$ (by 3 slides).

Proof. By a vertical slide we can guarantee continuum many non-empty horizontal lines. Then we can move them horizontally so that each vertical line contains at least one point, which implies that by vertically translating them we can cover $e$.

Claim 10. If $\operatorname{card}\left(\mathbb{R}^{2} \backslash H\right)=\mathfrak{c}$, then $H$ can be mapped into $e=\mathbb{R} \times\{0\}$ (by 13 slides).

Proof. By Claim 9 we can map $\mathbb{R}^{2} \backslash H$ to a set that contains $e$. Clearly, the same map maps $H$ into $\mathbb{R}^{2} \backslash e$. Then, by Claim 8 , we can map the image into $e$.

Claim 11. If $\operatorname{card}\left(\mathbb{R}^{2} \backslash H\right)=\mathfrak{c}$, then any permutation $p$ of $H$ can be realized by a fixed number $(27)$ of slides. (We say that a permutation $q$ of $\mathbb{R}^{2}$ "realizes" the permutation $p$ of $H \subset \mathbb{R}^{2}$ if $\left.q\right|_{H}=p$.)

Proof. Let $\phi$ be the map of Claim 10, which maps $H$ into $e$. By a vertical slide $V$ we can move the points of $\phi(H)$ to different horizontal lines. Then we can translate these lines horizontally, putting (for each $x \in H) V(\phi(x))$ above (or below) $\phi(p(x)$ ), then by a vertical slide to $\phi(p(x))$ and finally by $\phi^{-1}$ to $p(x)$.

Claim 12. If a permutation $p$ of $\mathbb{R}^{2}$ has continuum many fixed points, then it can be realized by a fixed number (105) of slides. 
Proof. Let $C=A \cup B$ be a partition of the set $C$ of fixed points such that $A$ and $B$ have cardinality $\mathfrak{c}$. By a theorem of Ore [2] any permutation $q$ of an infinite set can be written as $q=a^{-1} b^{-1} a b$, where $a$ and $b$ are permutations of the same set. Thus we can find permutations $a$ and $b$ of $\mathbb{R}^{2} \backslash C$ such that $\left.p\right|_{\mathbb{R}^{2} \backslash C}=a^{-1} b^{-1} a b$. Using Claim 11 (for $\mathbb{R}^{2} \backslash A$ and for $\mathbb{R}^{2} \backslash B$ ), there exists a map $\phi_{a}$ that realizes $a$ on $\mathbb{R}^{2} \backslash C$ and the identity on $A$, and a map $\phi_{b}$ that realizes $b$ on $\mathbb{R}^{2} \backslash C$ and the identity on $B$. Then $\psi=\phi_{a}^{-1} \phi_{b}^{-1} \phi_{a} \phi_{b}$ realizes $p$.

Claim 13. Any permutation of the plane can be realized by a fixed number (209) of slides.

Proof. Since every permutation consists of disjoint (finite or countable) cycles, any permutation of the plane can be written as a product of two permutations that have continuum many fixed points. Therefore Claim 12 completes the proof.

Remark 14. With a worse constant (417 instead of 209) one can prove Theorem 2 without using the theorem of Ore. Indeed, one can easily check that any permutation of the plane is the product of 4 permutations of order 2 such that each has an infinite support and continuum many fixed points. Then, using $(1,2)(3,4)=$ $(1,2,3)^{-1}(1,2,4)^{-1}(1,2,3)(1,2,4)$ one can easily write these 4 permutations as commutators of the same support, so the argument of Claim 12 can be used.

Remark 15. By a different (set-theoretic) method Péter Komjáth [3] improved our result showing that if a subset of the plane and its complement have continuum cardinality, then it can be mapped to $e=\mathbb{R} \times\{0\}$ by 5 slides. This means that in Claim 10 it is enough to use 5 slides, which implies that by 81 slides any permutation of the plane can be realized.

In fact, Komjáth's method works in any infinite Abelian group: he proved that for any infinite Abelian group $A$, if $B \subset A \times A$ and $\operatorname{card}(B)=\operatorname{card}((A \times A) \backslash B)$, then $B$ can be mapped to $A \times\{0\}$ by 5 slides. Note that after Claim 10 our method also works for any infinite Abelian group $A$ (if $A$ is countable, then in Claim 13 we also have to check that an infinite cycle can be written as the product of two permutations that have infinitely many fixed points). Therefore we get that for any infinite Abelian group $A$, any permutation of $A \times A$ is the composition of 81 slides. This also means that the full symmetric group acting on any infinite set is a product of 81 copies of two isomorphic Abelian subgroups.

The above results are not true for finite Abelian groups. First, for some Abelian groups (e.g. cyclic groups of odd order) any slide is an even permutation. However, one can show that for any Abelian group $A$ any even permutation of $A$ can be obtained as a composition of slides. But the number of slides one has to use is unbounded (see [1]).

Questions 16. It seems interesting to ask what happens if we make some natural restriction (e.g. continuous, measurable, Lipschitz, polynomial) about the functions in the definition of slides. For example what permutations of the plane can we get by finitely many "continuous" slides? Clearly we can get only orientation and measure preserving homeomorphisms but one can show that not all of them can be transformed: a continuous slide can make only a "bounded twist", so homeomorphisms with "unbounded twists" cannot be realized by finitely many continuous slides.

Clearly, "measurable" slides are measure preserving permutations of the plane. Are there other invariants besides the measure of the set and the measure of its 
complements? Can all measure preserving permutations of the plane be obtained by finitely many measurable slides?

\section{REFERENCES}

[1] M. Abért, Symmetric groups as products of Abelian subgroups, to appear in Bull. London Math. Soc.

[2] O. Ore, Some remarks on commutators, Proc. Amer. Math. Soc. 2 (1951), 307-314. MR 12:671e

[3] P. Komjáth, Five degrees of separation, to appear in Proc. Amer. Math. Soc.

Department of Algebra, Eötvös Loránd University, Kecskeméti u. 10-12, 1053 BuDAPEST, HUNGARY

E-mail address: abert@cs.elte.hu

Department of Analysis, Eötvös Loránd University, Kecskeméti u. 10-12, 1053 BuDAPEST, HUNGARY

E-mail address: elek@cs.elte.hu 